\title{
Stability Preserving Transformations of Graphs
}

\author{
Vadim V. Lozin \\ DIMAP and Mathematics Institute \\ University of Warwick \\ V.Lozin@warwick.ac.uk
}

\begin{abstract}
Graph transformations proved useful for many algorithmic problems. In the present paper, we study this tool with respect to the maximum stable set problem. We first review available results on this topic and then propose an approach to uniformly describe and systematically develop graph transformations that do not change the size of a maximum stable set in the graph. The approach is illustrated by a number of new transformations.
\end{abstract}

Keywords: Graph transformation; Stability number

\section{Introduction}

A subset of pairwise nonadjacent vertices in a graph $G$ is called stable (or independent). The cardinality of a maximum size stable set in $G$ is called the stability number of $G$ and is denoted $\alpha(G)$. The problem of determining a stable set of maximum cardinality finds important applications in various fields, including computer vision and pattern recognition. We refer to [6] for a review concerning algorithms, applications, and complexity issues of this problem.

A helpful tool to solve or simplify the maximum stable set problem is based on transforming a given graph $G$ into a new graph $G^{\prime}$ in such a way that the difference $\alpha\left(G^{\prime}\right)-\alpha(G)$ is easy to compute. A trivial example is given by the deletion of an isolated vertex, which reduces the stability number by exactly one. A more sophisticated example comes from matching theory and is known as the cycle shrinking (see e.g. [29]). This reduction is a key tool to solve the maximum matching problem, which is equivalent to the problem of finding a maximum stable set in a line graph.

The literature provides many more examples of graph transformations that can be useful for the maximum stable set problem. We give an overview on this topic in Section 2. Originally, these transformations have been described in different terms and have been obtained by different means. For instance, a good source of transformations preserving the stability number is the area of pseudo-Boolean optimization [7, 15]. Several interesting transformations of this type have been derived from Boolean arguments (see e.g. [24]). In the present paper, we exploit a purely graph-theoretic approach. Our purpose is to provide a uniform way to describe and develop graph transformations for the maximum stable set problem. To this end, we employ the notion of transformation plan, introduced 
in [2], and elaborate it in a non-trivial way. This elaboration leads to generalization of several previously known transformations.

\section{An overview}

Let us begin with the following simple introductory example. Assume a graph $G$ contains a vertex $x$ of degree 1 with the only neighbor $y$. Then, obviously, every stable set $S$ containing $y$ does not contain $x$, and hence, $y$ can be replaced in $S$ by $x$. Therefore, the removal of $y$ from the graph does not change its stability number. By means of this operation any graph can be transformed into a graph that does not contain vertices of degree 1 and has the same stability number as the initial graph. For some specific graphs, such as trees, this leads to an efficient algorithm to solve the maximum stable set problem. This simple reduction admits many interesting generalizations, some of which are listed below.

1. Simplicial vertex reduction. A vertex $x$ is said to be simplicial if the neighborhood of $x$ is a clique. For instance, every vertex of degree 1 is simplicial. With the arguments similar to those used in the above introductory example, one can easily show that $\alpha(G-y)=\alpha(G)$ for any vertex $y$ in the neighborhood of a simplicial vertex $x$.

This reduction provides a polynomial-time solution for the maximum stable set problem in the class of chordal graphs (a proper generalization of trees), as any chordal graph contains at least two simplicial vertices.

2. Neighborhood reduction. Let $x$ and $y$ be adjacent vertices in a graph $G$. Partition the remaining vertices of the graph into four subsets as follows (see Figure 1(a) for an illustration):

$c_{x}$, the subset of vertices adjacent to $x$ and nonadjacent to $y$,

$c_{y}$, the subset of vertices adjacent to $y$ and nonadjacent to $x$,

$c_{x y}$, the subset of vertices adjacent both to $x$ and to $y$,

$c_{\emptyset}$, the subset of vertices adjacent neither to $x$ nor to $y$.

If $c_{x}=\emptyset$, then for any stable set $S$ with $y \in S$, the set $(S-\{y\}) \cup\{x\}$ is stable too. Therefore, the removal of $y$ from the graph does not change its stability number. This reduction has been discovered independently by many researchers under various names such as neighborhood reduction or elementary compression. Notice that in the special case when $c_{x y}$ is a clique the neighborhood reduction coincides with the simplicial vertex reduction. In [28], the neighborhood reduction has been used to reduce any circular arc graph to a special canonical form which allows a simple solution to the stable set problem, thus providing an efficient algorithm to solve the problem in the class of circular arc graphs.

3. Magnet. As before, we consider two adjacent vertices $x$ and $y$ as represented in Figure 1(a). Assume that every vertex in $c_{x}$ is adjacent to every vertex in $c_{y}$. A pair of vertices $x, y$ with this property has been called in [21] a magnet. An important property of a magnet is that the removal of $y$ together with the edges of the form $x z$ with $z \in c_{x}$ does not change the stability number of the graph. We shall refer to this transformation as magnet simplification. Clearly the neighborhood reduction is a special case of the magnet 


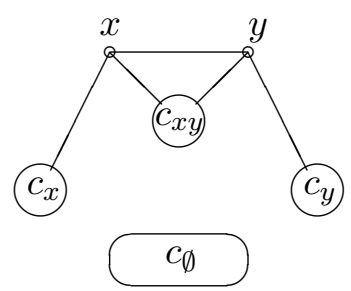

(a)

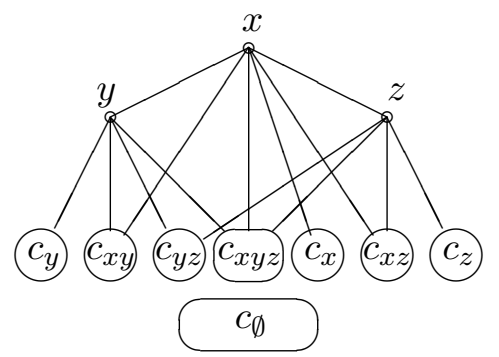

(b)

Figure 1: Partitioning a graph with respect to 2 (a) or 3 (b) vertices

simplification when $c_{x}$ is empty. Several interesting applications of the this reduction for the computation of the stability number can be found in [25].

4. Vertex deletion. Now let vertices $x, y$ and $z$ induce a path $P_{3}$ with edges $x y$ and $x z$. Partition the remaining vertices of the graph into eight subsets with respect to $x, y, z$ as shown in Figure 1(b). It has been observed in [5] that if $c_{y} \cup c_{z} \cup c_{y z}$ is a clique, then the removal of $x$ does not change the graph stability number.

5. Edge deletion. Several stability preserving transformations reducing the number of edges have been proposed in [8]. We describe a single example that deals with three vertices $x, y, z$ inducing a $P_{3}$ as shown in Figure 1(b). If $c_{y}=\emptyset$, then the removal of the edge $x z$ from the graph does not change its stability number.

It is interesting to notice that the magnet simplification (Example 3) can be obtained as a combination of the edge deletion and the neighborhood reduction. Indeed, if a pair of vertices $x, y$ forms a magnet in a graph, then for any vertex $z \in c_{x}$, every neighbor of $y$ is adjacent either to $x$ or to $z$ and hence the edge $x z$ can be deleted without changing the stability number of the graph. After deletion of all edges connecting $x$ to its private neighbors (i.e., to the vertices in $c_{x}$ ), the neighborhood reduction can be applied to the pair $x, y$.

6. BAT-reduction. Alain Hertz [24] defined a BAT in a graph to be a subset of three vertices $x, y, z$ with the following properties:

(a) vertices $x, y, z$ induce a $P_{3}$ as shown in Figure 1(b);

(b) each vertex in $c_{x}$ is adjacent to each vertex in $c_{y} \cup c_{y z} \cup c_{x y}$ or (not exclusively) to each vertex in $c_{z} \cup c_{y z} \cup c_{x z}$;

(c) each vertex in $c_{x z}$ is adjacent to each vertex in $c_{y} \cup c_{y z} \cup c_{x y}$;

(d) each vertex in $c_{x y}$ is adjacent to each vertex in $c_{z} \cup c_{y z} \cup c_{x z}$.

Given a graph $G=(V, E)$ and a BAT in $G$, we define $G^{\prime}=\left(V^{\prime}, E^{\prime}\right)$ as follows:

- the vertex set $V^{\prime}$ of $G^{\prime}$ is obtained from $V$ by removing the vertices $x, y, z$ and by adding two new vertices $a$ and $b$; 
- the edge set $E^{\prime}$ of $G^{\prime}$ is obtained from $E$ by removing all the edges incident to $x, y$ or $z$ and by linking $a$ to each vertex in $c_{x y z}$, and by linking $b$ to each vertex in $c_{y} \cup c_{x y} \cup c_{x y z} \cup c_{y z} \cup c_{x z} \cup c_{z}$.

It has been proved in [24] that $\alpha(G)=\alpha\left(G^{\prime}\right)$. We shall refer to the described transformation as the BAT-reduction.

7. Stability changing transformations can be viewed as a special case of stability preserving transformations. Indeed, if a certain transformation transforms a graph $G$ into a graph $G^{\prime}$ with $\alpha(G)=\alpha\left(G^{\prime}\right)-k$ for some integer $k$, then the addition of $k$ isolated vertices either to $G$ (if $k$ is positive) or to $G^{\prime}$ (if $k$ is negative) makes the transformation stability preserving.

Consider, for instance, the special case of the BAT-reduction with $c_{x}=c_{x y}=c_{x z}=$ $c_{x y z}=\emptyset$. In that case, the new vertex $a$ is isolated in the graph $G^{\prime}$ and hence the removal of $a$ from $G^{\prime}$ decreases its stability number by exactly one. As a result, we obtain the transformation illustrated in Figure 2:

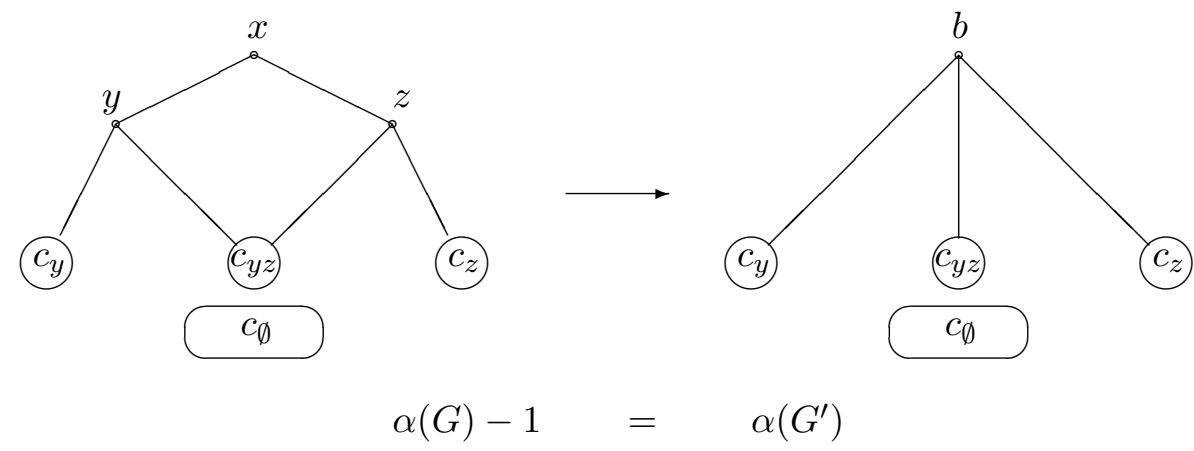

Figure 2: Vertex folding

This transformation is known as vertex folding. It was used to improve the worst case time complexity for the vertex cover and stable set problems [11, 17].

Notice that the transformation inverse to vertex folding is applicable to any graph. It was used in [1] under the name vertex splitting in order to reduce in polynomial time the maximum stable set problem from the class of all graphs to some restricted classes. A weaker version of vertex splitting was used in [34] to prove NP-hardness of the problem in graphs with large girth.

Now let us return to the vertex folding and observe that this transformation admits a wide generalization. Given a graph $G$ and a vertex $x$ in $G$, we transform $G$ by deleting $x$ together with all its neighbors and by introducing a new vertex for every pair of nonadjacent vertices in the neighborhood of $x$. With an appropriate connection of the new vertices to each other and to the vertices in the rest of the graph, this transformation reduces the stability number of the graph by exactly one. Repeated applications of this transformation lead to a general approach to compute the stability number of an arbitrary graph. This approach was proposed in [16] and was called there the struction method. Unfortunately, the number of vertices is generally increasing when the transformation is applied. However, specialized versions of the struction method have been designed to 
compute the stability number of graphs in particular classes in polynomial time [19, 20, 22]. Some restricted versions of the struction method have been applied to the maximum stable set problem in $[4,17]$. More on the struction method can be also found in [14, 27].

A generalization of the struction method has been proposed in [3]. It is based on a transformation which, given a graph $G$ and an induced subgraph $H$ of $G$, substitutes $H$ together with its neighbourhood by a new graph in such a way that the stability number of the resulting graph is exactly $\alpha(H)$ less that that of $G$. This generalization includes several particular graph transformations studied in the literature, such as

crown reduction (was used to prove fixed-parameter tractability of the minimum vertex cover problem [12]),

cycle shrinking (a key tool to solve the maximum matching problem, which is equivalent to the maximum stable set problem in the class of line graphs [29]),

clique reduction (was used to derive polynomial time algorithms for the maximum stable set problem in several particular classes of graphs [13, 26, 23]. Also, the edge projection, which is a specialization of the clique reduction when restricted to edges, has been used in $[32,33]$ to develop some heuristics for the stable set problem).

We refer the reader to $[2,10,30]$ for more examples of transformations preserving or changing the stability number by a constant.

8. Related transformations. Several examples of graph transformations helpful for the maximum stable set problem can be found in the literature devoted to some related problems. We mentioned already vertex folding and crown reduction that have been originally developed for the minimum vertex cover problem.

Another example can be found in [18] where the authors propose a transformation that preserves the clique number. One more example is known as the even pair contraction, an operation that has been used to solve the vertex coloring problem in some classes of perfect graph (see e.g. [31]). Since in a perfect graph $G$, the chromatic number equals the clique number, the same transformation solves the maximum clique problem in $G$, and hence, the maximum independent set problem in the complement of $G$.

\section{A transformation plan}

Having examined a number of particular examples in the previous section, we now turn to the problem of general description of graph transformations. To this end, we first propose a formalization of the notion of graph transformation. While we apply our transformations to simple graphs only, the formalism we introduce below involves the notion of a hypergraph, i.e., a pair $(V, \mathcal{E})$, where $V$ is a set of vertices and $\mathcal{E} \subseteq 2^{V}$ is a set of hyperedges. For a hyperedge $e \in \mathcal{E}$, we denote by $|e|$ the number of vertices in $e$. A hyperedge $e$ with $|e|=1$ will be called trivial. A subset of vertices of a hypergraph will be called stable if it does not contain nontrivial hyperedges.

For a (hyper)graph $G$, we denote by $V(G)$ and $E(G)$ the vertex set and the edge set of $G$, respectively. If $U \subseteq V(G)$ is a subset of vertices of a (hyper)graph $G$, then $G[U]$ denotes the subgraph of $G$ induced by $U$, i.e., the (hyper)graph with the vertex set $U$ and 
the edge set consisting of those edges of $G$ that are subsets of $U$. Also, by $G-U$ we denote the subgraph of $G$ induced by the set $V(G)-U$.

In a (hyper)graph $G$, a vertex $x$ will be called a neighbor of a vertex $y$ if there is a (hyper)edge containing both $x$ and $y$. By $N(x)$ we denote the neighborhood of $x$, i.e., the set of all neighbors of $x$. Also, if $U$ and $W$ are two subsets of vertices of $G$, then $N_{U}(x)=N(x) \cap U$ is the neighborhood of $x$ in the set $U, N(W)=\bigcup_{x \in W} N_{V(G)-W}(x)$ is the neighborhood of the subset $W$, and $N_{U}(W)=N(W) \cap U$ is the neighborhood of $W$ in the subset $U$. Finally, we let $\overline{N_{U}(W)}=U-N_{U}(W)$.

Let $Z=(C, \mathcal{E})$ be a hypergraph with a set of vertices $C$ and a set of hyperedges $\mathcal{E}$, and let $X$ and $Y$ be two hypergraphs such that

$$
\begin{gathered}
V(X) \cap V(Y)=C, \\
X[C]=Y[C]=Z, \\
|e|=2 \text { for any } e \in(E(X)-\mathcal{E}) \cup(E(Y)-\mathcal{E}) .
\end{gathered}
$$

Definition 1. An ordered pair of hypergraphs $(X, Y)$ satisfying (1)-(3) will be called a transformation plan with the center $Z=(C, \mathcal{E})$.

We shall say that a plan $\Sigma=(X, Y)$ is applicable to a simple graph $G$ if the following three conditions hold:

(1.1) $G$ contains a subset of vertices $A$ inducing a subgraph isomorphic to the graph $X-C$. For simplicity, we shall assume that $A=V(X)-C$. Also, we shall denote $B:=V(Y)-C$, and $R:=V(G)-A$.

(1.2) For any vertex $x \in R$, there is a vertex $c(x) \in C$, called the image of $x$, such that $N_{A}(c(x))=N_{A}(x)$. For a subset of vertices $P \subseteq R$, we denote $c(P)=\bigcup_{x \in P} c(x)$.

(1.3) For any subset $P \subseteq R$ with $|P|>1$, if $c(P) \in \mathcal{E}$, then $P$ is not a stable set in $G$.

Let $\Sigma$ be a plan applicable to a graph $G$. Application of $\Sigma$ to $G$ consists of the following three steps:

(2.1) Delete from $G$ the set of vertices $A$ (together with all incident edges).

(2.2) Add to the remainder $G[R]$ of $G$ the graph $Y[B]$.

(2.3) For every vertex $x \in R$, link $x$ to each vertex in $N_{B}(c(x))$ by an edge.

A graph obtained from $G$ by application of a plan $\Sigma$ will be denoted $G^{\Sigma}$. Observe that there may exist different graphs $G^{\Sigma}$ obtained from $G$ by application of $\Sigma$.

Example. Let $\Sigma=(X, Y)$ be a transformation plan described by two graphs $X$ and $Y$ shown in Figure 3. The center of this plan is the graph induced by four vertices $c_{x}, c_{y}, c_{x y}, c_{\emptyset}$. The only edge of the center is $\left\{c_{x}, c_{y}\right\}$. It is not difficult to see that this plan describes the magnet simplification. 


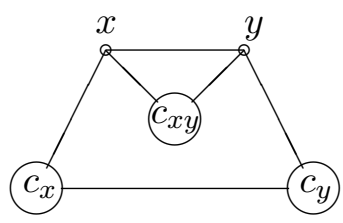

(CD)

$X$

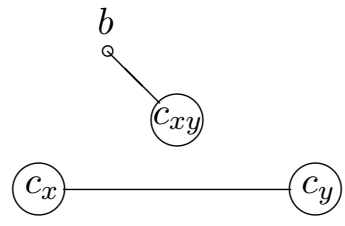

(CD)

$Y$

Figure 3: Graphs $X$ and $Y$ for the magnet simplification

\section{$4 \alpha$-preserving transformation plans}

In this section, we characterize those transformation plans that preserve the stability number.

Definition 2. A plan $\Sigma$ will be called $\alpha$-preserving if $\alpha(G)=\alpha\left(G^{\Sigma}\right)$ for any graph $G^{\Sigma}$ obtained from a graph $G$ by application of the plan $\Sigma$.

Theorem 1. Let $\Sigma=(X, Y)$ be a transformation plan with a center $Z=(C, \mathcal{E})$, and let $A=V(X)-C, B=V(Y)-C$. Then $\Sigma$ is $\alpha$-preserving if and only if

$$
\alpha\left(X\left[\overline{N_{A}\left(T_{0}\right)} \cup T_{1}\right]\right)=\alpha\left(Y\left[\overline{N_{B}\left(T_{0}\right)} \cup T_{1}\right]\right)
$$

for any stable set $T$ in the graph $Z$, where $T_{1}=\{x \in T:\{x\} \in \mathcal{E}\}$ and $T_{0}=T-T_{1}$.

Proof. In order to prove the sufficiency, let us consider a plan $\Sigma$ satisfying (4), and let $G^{\Sigma}$ be a graph obtained from a graph $G$ by application of $\Sigma$. Consider a stable set $S$ in the graph $G$, and denote $S_{A}:=S \cap A$ and $P:=S-S_{A}$. Since $P \subseteq R$, we can determine the set $T=c(P)$. Let us show that $T$ is a stable set in the graph $Z$. Indeed, if $T$ contains a nontrivial hyperedge $e$, then the set of preimages for $e$ in $P$ consists of more than one vertex and hence, by (1.3), is not a stable set in $G$, which contradicts the definition of $P$. Taking into account (1.2), we can also conclude that the set $S_{A} \cup T$ is stable in $X$.

The partition $T=T_{0} \cup T_{1}$ induces a respective partition $P=P_{0} \cup P_{1}$, i.e., $T_{i}=c\left(P_{i}\right)$ $(i=1,2)$. Let $x$ and $y$ be two distinct vertices in $P_{1}$. Since $c(x)$ and $c(y)$ belong to $T_{1}$, we conclude that $\{c(x)\}$ and $\{c(y)\}$ are hyperedges in $Z$. Therefore, $c(x) \neq c(y)$, otherwise $x$ would be adjacent to $y$ in $G$ (by (1.3)). Consequently,

$$
\left|P_{1}\right|=\left|T_{1}\right| .
$$

Denote $U:=\overline{N_{A}\left(T_{0}\right)} \cup T_{1}$ and $W:=\overline{N_{B}\left(T_{0}\right)} \cup T_{1}$. Clearly $S_{A} \cup T_{1} \subseteq U$. By the assumption, $\alpha(X[U])=\alpha(Y[W])$. Therefore, $W$ contains a subset of vertices $F$ which is stable in $Y$ and has cardinality

$$
|F|=\left|S_{A} \cup T_{1}\right| .
$$

Denote $F_{B}:=F \cap B, F_{1}:=F \cup T_{1}$. And let $P_{1}^{F}$ be the set of preimages for $F_{1}$ in $P_{1}$, i.e., $c\left(P_{1}^{F}\right)=F_{1}$. As before, it follows from (1.3) that

$$
\left|P_{1}^{F}\right|=\left|F_{1}\right| \text {. }
$$


By the construction, $P_{0} \cup P_{1}^{F} \cup F_{B}$ is a stable set in $G^{\Sigma}$. Taking into account (5)-(7), we conclude that its cardinality is

$$
\left|P_{0} \cup P_{1}^{F} \cup F_{B}\right|=\left|P_{0} \cup F_{1} \cup F_{B}\right|=\left|P_{0} \cup F\right|=\left|P_{0} \cup S_{A} \cup T_{1}\right|=\left|P_{0} \cup S_{A} \cup P_{1}\right|=|S| .
$$

Therefore, $\alpha(G) \leq \alpha\left(G^{\Sigma}\right)$. The inverse inequality can be proved in a similar way. Thus, $\alpha(G)=\alpha\left(G^{\Sigma}\right)$.

To prove the necessity, consider a stable set $T=\left\{c_{1}, \ldots, c_{k}, c_{k+1}, \ldots, c_{l}\right\}$ in the graph $Z$ with $c_{1}, \ldots, c_{k} \in T_{1}$ and $c_{k+1}, \ldots, c_{l} \in T_{0}$. Let $G$ be a graph with the vertex set $A \cup D_{1} \cup \ldots \cup D_{l}$, where $\left|D_{1}\right|=\cdots=\left|D_{k}\right|=1$ and $\left|D_{k+1}\right|=\cdots=\left|D_{l}\right|>\max \{|A|,|B|\}$, and the edge set consisting of all edges of the graph $X[A]$ and the edges linking every vertex in $D_{i}$ to every vertex in $N_{A}\left(c_{i}\right)(i=1, \ldots, l)$. Clearly the plan $\Sigma$ is applicable to the graph $G$.

Denote $D^{1}:=D_{1} \cup \ldots \cup D_{k}$ and $D^{0}:=D_{k+1} \cup \ldots \cup D_{l}$. Since $D_{i}>|A|$ for $i>k$, no maximum stable set in the graph $G$ contains a vertex in $N_{A}\left(D^{0}\right)$. Hence, every maximum stable set in $G$ is of the form $D^{0} \cup P$, where $P$ is a maximum stable set in the graph $G^{*}=$ $G\left[\overline{N_{A}\left(D^{0}\right)} \cup D^{1}\right]$. Since $N_{A}\left(D_{i}\right)=N_{A}\left(c_{i}\right)$ for $i=1, \ldots, l$ and $\left|D_{i}\right|=1$ for $i=1, \ldots, k$, it is not hard to see that the graph $G^{*}$ is isomorphic to the graph $X^{*}=X\left[\overline{N_{A}\left(T_{0}\right)} \cup T_{1}\right]$. Therefore, $\alpha\left(X^{*}\right)=\alpha\left(G^{*}\right)=|P|$.

Now let $G^{\Sigma}$ be a graph obtained from $G$ by application of the plan $\Sigma$. Similarly, every maximum stable set in $G^{\Sigma}$ is of the form $D^{0} \cup P^{\prime}$, where $P^{\prime}$ is a maximum stable set in the graph $G^{\Sigma *}=G^{\Sigma}\left[\overline{N_{B}\left(D^{0}\right)} \cup D^{1}\right]$. As before, the graph $G^{\Sigma *}$ is isomorphic to the graph $Y^{*}=Y\left[\overline{N_{B}\left(T_{0}\right)} \cup T_{1}\right]$. Therefore, $\alpha\left(Y^{*}\right)=\alpha\left(G^{\Sigma *}\right)=\left|P^{\prime}\right|$. By the assumption, $\alpha(G)=\alpha\left(G^{\Sigma}\right)$, which implies $|P|=\left|P^{\prime}\right|$, and therefore, $\alpha\left(X^{*}\right)=\alpha\left(Y^{*}\right)$.

\section{Development of stability preserving transformations}

In this section, we show how the results of Theorem 1 can be used to create new stability preserving transformations. In order to construct a plan $\Sigma=(X, Y)$, we start from graphs $X[A]$ and $Y[B]$ and then proceed to the center $Z=(C, \mathcal{E})$ and to the two sets of edges $E(A, C)=\{a c \in E(X): a \in A, c \in C\}$ and $E(B, C)=\{b c \in E(Y): b \in B, c \in C\}$.

It follows from Theorem 1 that for any $\alpha$-preserving plan, $\alpha(X[A])=\alpha(Y[B])$. This is the only restriction on the graphs $X[A]$ and $Y[B]$. Therefore, we may choose any two graphs with the same stability number as $X[A]$ and $Y[B]$. A plan with the given $X[A]$ and $Y[B]$ will be called an $(A, B)$-plan.

Example. For an illustration, let $X[A]$ be the graph with the vertex set $A=\{x, y, z\}$ and the edge set $\{x y, x z\}$, and $Y[B]$ be the graph with the vertex set $B=\{a, b\}$ and with no edges. Clearly, $\alpha(X[A])=\alpha(Y[B])$.

For simplicity of the illustration, we shall assume that for any subset $P \subseteq A$, there is at most one vertex of $C$, denoted $c_{P}$, with $N_{A}\left(c_{P}\right)=P$. In this case, the set of edges $E(A, C)$ is completely determined by the set of vertices $C$. Therefore, to construct an $\alpha$-preserving $(A, B)$-plan $\Sigma$, we only have to construct the graph $Z=(C, \mathcal{E})$, and the set of edges $E(B, C)$.

For the construction of $Z=(C, \mathcal{E})$, the following observation is useful. Let $\Sigma_{1}$ and $\Sigma_{2}$ be two $(A, B)$-plans with centers $Z_{1}=\left(C_{1}, \mathcal{E}_{1}\right)$ and $Z_{2}=\left(C_{2}, \mathcal{E}_{2}\right)$, respectively. It is not 
hard to see that if $C_{2} \subseteq C_{1}$ and $\mathcal{E}_{1} \subseteq \mathcal{E}_{2}$, then the applicability of $\Sigma_{2}$ to a graph $G$ implies the applicability of $\Sigma_{1}$ to $G$. Therefore, in order to construct an $(A, B)$-plan with the largest applicability, we start with $\mathcal{E}$ being empty and $C$ being maximal, i.e. we assume that for any subset $P \subseteq A$, the set $C$ contains a vertex $c_{P}$ with $N_{A}\left(c_{P}\right)=P$. The process of the creation of the plan now reduces to determining the set $E(B, C)$ and revising the sets $C$ and $\mathcal{E}$, if necessary.

Example continued. As instructed above, we start with $C:=\left\{c_{\emptyset}, c_{x}, c_{y}, c_{z}, c_{x y}, c_{x z}\right.$, $\left.c_{y z}, c_{x y z}\right\}$, and $\mathcal{E}:=\emptyset$.

Now we proceed to the set of edges $E(B, C)$. Any choice of this set leads to a valid transformation plan. However, some choices may require revisions of the sets $C$ and $\mathcal{E}$. Indeed, by Theorem 1 , for any vertex $c \in C$, there must be valid either the equality

$$
\alpha\left(X\left[A-N_{A}(c)\right]\right)=\alpha\left(Y\left[B-N_{B}(c)\right]\right)
$$

if $\{c\} \notin \mathcal{E}$, or the equality

$$
\alpha(X[A \cup\{c\}])=\alpha(Y[B \cup\{c\}])
$$

if $\{c\} \in \mathcal{E}$.

Therefore, if the set $E(B, C)$ is chosen so that neither (8) nor (9) is valid for some vertex $c \in C$, then $c$ must be excluded from $C$. If (9) is valid but (8) is not, then $\{c\}$ must be included in the set $\mathcal{E}$ as a trivial hyperedge. Observe that validity of (8) implies validity of (9). Indeed, $\alpha(X[A \cup\{c\}])=\max \left\{\alpha(X[A]), \alpha\left(X\left[A-N_{A}(c)\right]\right)+1\right\}$, and similarly, $\alpha(Y[B \cup\{c\}])=\max \left\{\alpha(Y[B]), \alpha\left(Y\left[B-N_{B}(c)\right]\right)+1\right\}$. By the assumption, $\alpha(X[A])=\alpha(Y[B])$. Therefore, (8) implies (9).

Example continued. We propose the following two possible solutions for the set $E(B, C)$ leading to two different transformation plans, which we distinguish by indexing the respective sets where necessary:

$$
\begin{gathered}
E_{1}(B, C)=\left\{a c_{x y z}, b c_{y}, b c_{x y}, b c_{x y z}, b c_{y z}, b c_{x z}, b c_{z}\right\} . \\
E_{2}(B, C)=\left\{a c_{x y z}, a c_{y}, a c_{x y}, a c_{y z}, b c_{x y z}, b c_{y z}, b c_{x z}, b c_{z}\right\} .
\end{gathered}
$$

In the first plan, equality (8) is satisfied for each vertex in $C$, and hence neither $C$ nor $\mathcal{E}_{1}$ is revised at this stage.

In the second plan, equality (8) is not satisfied for $c_{y z}$. However, in this case equality (9) is valid. Therefore, in the second plan we do not modify the set $C$ but we must include $\left\{c_{y z}\right\}$ as a trivial hyperedge in the set $\mathcal{E}_{2}$.

In the last stage of the procedure, we have to determine nontrivial hyperedges in $\mathcal{E}$. Starting with $k=2$, we verify equality (4) for all stable sets $T$ of cardinality $k$ in the current graph $Z=(C, \mathcal{E})$. If $T$ does not satisfy (4), it must be included in $\mathcal{E}$ as a hyperedge. After the inspection of all stable sets of cardinality $k$, we proceed to stable sets of cardinality $k+1$, and so on. 
Example continued. Following the instructions, we conclude that

$$
\begin{gathered}
\mathcal{E}_{1}=\left\{\left\{c_{x y}, c_{y z}\right\},\left\{c_{x y}, c_{z}\right\},\left\{c_{x y}, c_{x z}\right\},\left\{c_{y z}, c_{x z}\right\},\left\{c_{x z}, c_{y}\right\},\left\{c_{x}, c_{y z}\right\},\left\{c_{x}, c_{y}, c_{z}\right\}\right\} . \\
\mathcal{E}_{2}=\left\{\left\{c_{y z}\right\},\left\{c_{y z}, c_{y}\right\},\left\{c_{y z}, c_{z}\right\},\left\{c_{y}, c_{z}\right\}\right\} .
\end{gathered}
$$

This completes the construction of two transformations plans.

It is not hard to verify that the first of the two constructed plans generalizes the BAT-reduction (Example 6 of Section 2). Indeed, according to the structure of the graphs $X[A], Y[B]$ and the set $E_{1}(B, C)$, application of the first plan consists of the same steps as the BAT-reduction. However, the class of graphs to which this plan is applicable is wider than the class of graphs containing a BAT. The condition (a) in the definition of BAT is clearly satisfied by the choice of $X[A]$, the condition (d) corresponds to the first three hyperedges in the set $\mathcal{E}_{1}$, the condition (c) corresponds to the hyperedges $3,4,5$ in $\mathcal{E}_{1}$. The requirement that every vertex in $c_{x}$ must be adjacent to every vertex in $c_{y z}$ (condition (b) in the definition of BAT) corresponds to the edge $\left\{c_{x}, c_{y z}\right\} \in \mathcal{E}_{1}$, while the rest of condition (b) is a particular case of the requirement provided by the hyperedge $\left\{c_{x}, c_{y}, c_{z}\right\} \in \mathcal{E}_{1}$. Indeed, this edge does not allow three vertices $u \in c_{x}, v \in c_{y}$ and $w \in c_{z}$ form a stable set. Clearly this requirement is satisfied whenever condition (b) holds. But the converse is generally not true.

Similarly, one can verify that the second plan constructed in this section is a generalization of Example 4 of Section 2. It generalizes Example 4 in the sense that $c_{y}$ and $c_{z}$ need not to be cliques anymore.

\section{Conclusion}

In this paper, we proposed a formalization for the notion of graph transformation and characterized those transformations that preserve the stability number. This formalization allows to develop new stability preserving transformations in a systematic way. New transformations can be used to distinguish larger classes of graphs with polynomial-time solvable stable set problem or faster algorithms for this problem in general graphs. They can also be used to compute better combinatorial bounds on the size of a maximum stable set. Taking into account close relationship of the graph stability problem with pseudoBoolean optimization, we also believe that the results obtained in this paper will find applications far beyond graph theory.

\section{References}

[1] V.E.Alekseev, On the local restrictions effect on the complexity of finding the graph independence number, Combinatorial-algebraic methods in applied mathematics, Gorkiy Univ. Press, Gorkiy, (1983), 3-13 (in Russian).

[2] V.E. Alekseev and V.V. Lozin, Local transformations of graphs preserving independence number, Discrete Applied Mathematics, 135 (2004) 17-30. 
[3] G. Alexe, P.L. Hammer, V.V. Lozin and D. De Werra, Struction revisited, Discrete Applied Mathematics, 132 (2003) 27-46.

[4] R. Beigel, Finding maximum independent sets in sparse and general graphs, Proceedings of the 10th ACM-SIAM Symposium on Discrete Algorithms (SODA 1999), 856-857.

[5] A. Billionnet, Reductions and optimality conditions in the problem of a maximum weighted stable set, RAIRO Rech. Oper. 15 (1981), no. 3, 213-231 (in French).

[6] I.M. Bomze, M. Budinich, P.M. Pardalos and M. Pelillo, The maximum clique problem, in Handbook of Combinatorial Optimization - Suppl. Vol. A, D.-Z. Du and P.M. Pardalos, eds., Kluwer Academic Publishers, Boston, MA, 1999, 1-74.

[7] E. Boros and P.L. Hammer, Pseudo-Boolean optimization, Discrete Appl. Math. 123 (2002) 155-225.

[8] L. Butz, P.L. Hammer and D. Haussmann, Reduction methods for the vertex packing problem, Proceedings of the 17th Conference on Probability Theory (Brasov 1982), 73-79, VNU Sci. Press, Utrecht, 1985.

[9] A. BrandstäDt and P.L. Hammer, On the stability number of claw-free $P_{5}$-free and more general graphs, Discrete Applied Math. 95 (1999) 163-167.

[10] A. BrandstäDt and V.V. LozIn, A note on $\alpha$-redundant vertices in graphs, Discrete Applied Math. 108 (2001) 301-308.

[11] J. Chen, I. A. Kanj, and W. Jia, Vertex Cover: Further Observations and Further Improvements, Journal of Algorithms, 41 (2001) 280-301.

[12] R.G. Downey and M.R. Fellows, Parameterized complexity, Monographs in Computer Science, Springer-Verlag, New York, 1999.

[13] C. De Simone and A. Sassano, Stability number of bull- and chair-free graphs, Discrete Appl. Math. 41 (1993) 121-129.

[14] D. DE WERrA, On some properties of the struction of the graph, SIAM J. Algebra Discrete Methods, 5 (1984) 239-243.

[15] D. De Werra and P.L. Hammer, Weighted stability number of graphs and weighted satisfiability: The two facets of pseudo-Boolean optimization, Annals of Operations Research, 149 (2007) 67-73.

[16] Ch. Ebenegger, P.L. Hammer and D. De Werra, Pseudo-Boolean Functions and Stability of Graphs, Ann. Discrete Math. 19 (1984) 83-98.

[17] F. Fomin, F. Grandoni and D. Kratsch, Measure and Conquer: A Simple $O\left(2^{0.288 n}\right)$ Independent Set Algorithm, Proceedings of SODA 2006, 18-25.

[18] M.U. Gerber and A. Hertz, A transformation which preserves the clique number, J. Combin. Theory Ser. B 83 (2001) 320-330. 
[19] P.L. Hammer, N.V.R. MahadeV and D. DE Werra, Stability in CAN-free graphs, J. Combin. Theory Ser. B 38 (1985) 23-30.

[20] P.L. Hammer, N.V.R. Mahadev and D. De Werra, The struction of a graph: application to CN-free graphs, Combinatorica, 5 (1985) 141-147.

[21] P.L. Hammer and A. Hertz, On a transformation which preserves the stability number, RUTCOR Research report 69-91, Rutgers University, 1991.

[22] A. Hertz, Quelques utilisations de la struction, Discrete Math. 59 (1986) 79-89.

[23] A. Hertz, Polynomially solvable cases for the maximum stable set problem, Discrete Applied Math. 60 (1995) 195-210.

[24] A. Hertz, On the use of Boolean methods for the computation of the stability number, Discrete Applied Math. 76 (1997) 183-203.

[25] A. Hertz, On a graph transformation which preserves the stability number, Yugoslav Journal of Operations Research, 10/1 (2000), 1-12.

[26] A. Hertz and D. De Werra, On the stability number of $A H$-free graphs, J. Graph Theory, 17 (1993) 56-63.

[27] K.W. Hoke and M.F. Troyon, The struction algorithm for the maximum stable set problem revisited, Discrete Mathematics, 131 (1994) 105-113.

[28] M.C. Golumbic and P.L. Hammer, Stability in circular arc graphs, J. Algorithms, 9 (1988) 56-63.

[29] L. Lovász and M.D. Plummer, Matching theory, Ann. Discrete Math. 29 (1986) 471-480.

[30] V.V. Lozin, Conic reduction of graphs for the stable set problem, Discrete Math. 222 (2000) 199-211.

[31] F. Maffray and N. Trotignon, A class of perfectly contractile graphs, J. Combin. Theory Ser. B 96 (2006) 1-19.

[32] C. Mannino and A. Sassano, Edge projection and the maximum cardinality stable set problem, DIMACS Series in Discrete Mathematics and Theoretical Computer Science, 26 (1996) 205-219.

[33] C. Mannino and E. Stefanutti, An augmentation algorithm for the maximum weighted stable set problem, Computational Optimization and Applications, 14 (1999) 367-381.

[34] O.J. Murphy, Computing independent sets in graphs with large girth, Discrete Applied Mathematics, 35 (1992) $167-170$. 